\title{
BLM SUMOylation regulates ssDNA accumulation at stalled replication forks
}

\author{
Karen J. Ouyang ${ }^{1+}$, Mary K. Yagle ${ }^{2}$, Michael J. Matunis ${ }^{3}$ and Nathan A. Ellis ${ }^{2 *}$ \\ ${ }^{1}$ Department of Medicine, University of Chicago, Chicago, IL, USA \\ ${ }^{2}$ Department of Pediatrics, Institute of Human Genetics, University of Illinois at Chicago, Chicago, IL, USA \\ ${ }^{3}$ Department of Biochemistry and Molecular Biology, Bloomberg School of Public Health, Johns Hopkins University, Baltimore, MD, USA
}

\section{Edited by:}

Antonio Porro, Ecole Polytechnique

Federale de Lausanne, Switzerland

Reviewed by:

Graham Dellaire, Dalhousie

University, Canada

Julia Sidorova, University of

Washington, USA

Nathan A. Ellis, Department of

Pediatrics, Institute of Human

Genetics, University of Illinois at

Chicago, 900 South Ashland

Avenue, MC 767, Chicago, IL 60607,

USA

e-mail:naellis@uic.edu

${ }^{\dagger}$ Present address:

Karen J. Ouyang, Department of

Medical and Molecular Genetics,

Indiana University School of

Medicine, Indianapolis, USA

\section{*Correspondence:}

Polymerase stalling results in uncoupling of DNA polymerase and the replicative helicase, which generates single-stranded DNA (ssDNA). After stalling, RAD51 accumulates at stalled replication forks to stabilize the fork and to repair by homologous recombination (HR) double-strand breaks (DSBs) that accumulate there. We showed recently that SUMO modification of the BLM helicase is required in order for RAD51 to accumulate at stalled forks. In order to investigate how BLM SUMOylation controls RAD51 accumulation, we characterized the function of HR proteins and SSDNA-binding protein RPA in cells that stably expressed either normal BLM $(B L M+)$ or SUMO-mutant BLM (SM-BLM). In HU-treated SM-BLM cells, mediators BRCA2 and RAD52, which normally substitute RAD51 for RPA on SsDNA, failed to accumulate normally at stalled forks; instead, excess RPA accumulated. SM-BLM cells also exhibited higher levels of HU-induced chromatin-bound RPA than BLM+cells did. The excess RPA did not result from excessive intrinsic BLM helicase activity, because in vitro SUMOylated BLM unwound similar amounts of replication-fork substrate as unSUMOylated BLM. Nor did BLM SUMOylation inhibit binding of RPA to BLM in vitro; however, in immunoprecipitation experiments, more BLM-RPA complex formed in HU-treated SM-BLM cells, indicating that BLM SUMOylation controls the amount of BLM-RPA complex normally formed at stalled forks. Together, these results showed that BLM SUMOylation regulates the amount of ssDNA that accumulates during polymerase stalling. We conclude that BLM SUMOylation functions as a licensing mechanism that permits and regulates HR at damaged replication forks.

Keywords: Bloom's syndrome, DNA repair foci, homologous recombination, RecQ DNA helicases, replication fork stability

\section{INTRODUCTION}

DNA replication is a fundamental process in all living organisms in which the genetic material is duplicated. Highly regulated checkpoint and DNA repair mechanisms ensure that the genome is faithfully replicated with each round of cell division. In mammalian cells, homologous recombination (HR) is an essential repair mechanism that stabilizes damaged DNA replication forks, repairs double-strand breaks (DSBs) that occur during DNA replication, and helps restore productive DNA synthesis following disruption or breakage of replication forks. Although HR is critical in maintaining genome integrity during replication, it is tightly regulated to avoid harmful outcomes.

In the autosomal recessive, clinical entity Bloom's syndrome (BS), genome integrity is strikingly destabilized due to null mutations in the gene BLM (Ellis et al., 1995). The BLM protein is an ATP-dependent DNA helicase of the RecQ family, and it possesses multiple functions in DNA replication and HR (Lambert et al., 2010; Wechsler et al., 2011). BLM is one of the first components to be recruited to sites of DNA replication after treatment with agents that inhibit fork progression where it is thought to stabilize stalled forks (Davalos and Campisi, 2003; Sengupta et al., 2003, 2004). In HR-mediated DSB repair, BLM together with exonucleases EXO1 and DNA2 promote resection of DSBs, generating $3^{\prime}$ single-stranded DNA (ssDNA) tails that provide a substrate for loading of the RAD51 recombinase (Nimonkar et al., 2008, 2011; Mimitou and Symington, 2009). BLM preferentially unwinds substrates that resemble recombination intermediates, such as X-junctions and D-loops, and it is a member of a complex, which includes TopIII $\alpha$, BLAP75, and BLAP18 that possesses the unique capacity to dissolve a late recombination intermediate, the double Holliday junction, such that only non-crossover products are generated (Wu and Hickson, 2003; Raynard et al., 2006; Wu et al., 2006; Bussen et al., 2007; Singh et al., 2008; Xu et al., 2008). This activity is also important at sites of termination of DNA replication, because BLM accumulates on late replication intermediates to assist in duplex separation so that DNA replication can be efficiently completed (Chan et al., 2007, 2009; Lukas et al., 2011; Barefield and Karlseder, 2012).

BS cells, which lack BLM activity, display numerous characteristics that are the consequence of excessive HR, including high rates of loss of heterozygosity (Langlois et al., 1989; Groden et al., 1990; LaRocque et al., 2011), excessive chromosome abnormalities, such as telomere fusions, ring chromosomes, and quadriradial chromosomes (German, 1964; German and Crippa, 1966), and a high rate of sister chromatid exchange 
(SCE)(Chaganti et al., 1974). In addition, BS cells exhibit defects in DNA replication that might lead to excess $H R$, including accumulation of abnormal DNA replication intermediates (Lönn et al., 1990; Li et al., 2004), slower than normal DNA-chain growth (Hand and German, 1975; Rao et al., 2007), and abnormal origin firing (Davies et al., 2007). BS cells are hypersensitive to replication inhibitors such as hydroxyurea (HU)(Davies et al., 2004), and replication forks in BS cells recover inefficiently from HU-induced stalling, exhibiting accelerated accumulation of DSBs after release (Davies et al., 2007; Ouyang et al., 2009; Sidorova et al., 2013).

In our previous work, we showed that BLM undergoes posttranslational modification at lysines K317 and K331 by SUMO-1 and SUMO-2 (Eladad et al., 2005); cells that expressed a GFPBLM that was mutated at these two SUMO-acceptor sites, which we refer to as SUMO-mutant BLM (SM-BLM) cells, exhibited impairments of replication-associated $\mathrm{HR}$, as evidenced by increased HU-induced DNA damage and reduced HU-induced SCE (Ouyang et al., 2009). We further showed that SM-BLM cells have a defect in the recruitment or retention of the RAD51 recombinase at stalled replication forks and that SUMOylation of BLM in vitro increased the binding efficiency between BLM and RAD51, suggesting that BLM SUMOylation could act as a switch to turn on BLM's function in HR repair of stalled forks (Ouyang et al., 2009). Although our data indicates that SUMOylation of BLM regulates the recruitment or retention of RAD51 at stalled replication forks, the mechanism underlying the defect in RAD51 localization in SM-BLM cells remains unclear.

Replication inhibitors such as HU cause stalling of replicative polymerases on DNA, uncoupling of the replicative helicase from polymerases, and the generation of excess ssDNA (Byun et al., 2005). After HU treatment, excess ssDNA is detectable within minutes as evidenced by the accumulation of focal concentrations of ssDNA binding protein-replication protein A (RPA) at stalled replication forks (Balajee and Geard, 2004; Petermann et al., 2010). In the HR pathway, RAD51 is normally loaded onto RPA-bound ssDNA by a process that involves mediators (e.g., BRCA2 and RAD52) that substitute RAD51 for RPA on ssDNA (Heyer et al., 2010); however, after treatment of normal cells with
HU, RAD51 does not accumulate at stalled replication forks for several hours (Saintigny et al., 2001; Petermann et al., 2010). We hypothesize that a licensing mechanism exists that controls the loading of RAD51 onto ssDNA at stalled replication forks. The licensing mechanism could prevent premature loading of RAD51 at stalled replication forks and activate HR after fork breakage. To explain the deficit of RAD51 loading at stalled forks in SM-BLM cells, we hypothesized that SUMOylation of BLM is a key step in the licensing mechanism. To test this hypothesis, we analyzed the function of mediators and RPA at stalled forks in HU-treated SM-BLM cells.

\section{RESULTS \\ MEDIATOR ACCUMULATION AT STALLED REPLICATION FORKS IS IMPAIRED IN SUMO-MUTANT BLM CELLS}

Because the loading mechanism of RAD51 onto RPA-bound ssDNA requires recruitment of mediators to the repair site (Thorslund et al., 2010), we tested whether the mediators BRCA2 and RAD52 localized normally in HU-treated SM-BLM cells. As expected, treatment of BLM+ cells with $0.5 \mathrm{mM} \mathrm{HU}$ for $24 \mathrm{~h}$ induced an increase in BRCA2 foci from 10.4 foci/cell to 22.9 foci/cell. On the contrary, despite the presence of approximately twice the number of $\gamma-\mathrm{H} 2 \mathrm{AX}$ and BLM foci, untreated SM-BLM cells exhibited 14.4 BRCA2 foci/cell and HU treatment failed to induce a significant increase in BRCA2 foci to 16.4 foci/cell (Figure 1A). Similarly, whereas HU treatment of BLM+ cells induced an increase in RAD52 foci from 22.6 foci/cell to 35.1 foci/cell, HU treatment of SM-BLM cells resulted in virtually no change in RAD52 foci (from 26.4 foci/cell in untreated cells to 24.9 foci/cell in HU-treated cells; Figure 1B). These data suggested that the impairment of RAD51 localization in SMBLM cells was explained by an upstream defect in mediator accumulation at stalled forks.

\section{EXCESS RPA ACCUMULATES AT STALLED REPLICATION FORKS IN SUMO-MUTANT BLM CELLS}

Because accumulation of BRCA2 and RAD52 mediators at stalled forks was impaired after $\mathrm{HU}$ treatment, we reasoned that the impairment could be caused by less ssDNA accumulation after
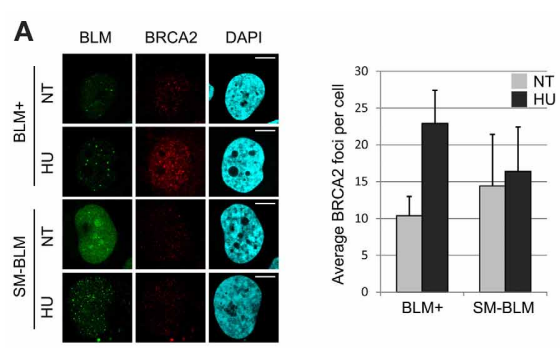

FIGURE 1 | Mediator accumulation at stalled replication forks is impaired in SUMO-mutant BLM cells. (A) Immunofluorescence images of BLM+ and SM-BLM cells untreated (NT) or treated with $0.5 \mathrm{mM} H U$ for $24 \mathrm{~h}(\mathrm{HU})$ and stained for BRCA2, with graphical representation of mean numbers of BRCA2 foci. (B) Same as (A) but stained for RAD52, with graphical representation as in (A). Two independent experiments were carried out on each of two BLM+ and
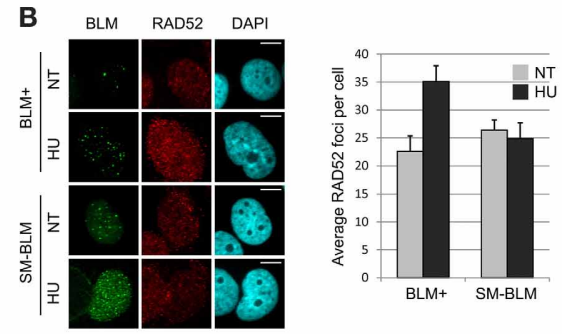

two SM-BLM clones. Error bars represent the standard deviations of the results of each experiment. After treatment with $\mathrm{HU}$, there were significantly more BRCA2 and RAD52 foci in BLM+ cells $(p<0.001$ for both comparisons) but not in SM-BLM cells ( $p=0.64$ for BRCA2 and $p=0.38$ for RAD52). $P$-values were calculated using mixed effects linear models as described in Materials and Methods. Bars indicate $10 \mu \mathrm{m}$. 
HU treatment. To test this possibility, we compared the accumulation of RPA at stalled forks in SM-BLM and BLM+ cells. We treated cells with or without $0.5 \mathrm{mM} \mathrm{HU}$ for $24 \mathrm{~h}$, then quantified RPA foci by scoring the number of RPA foci/cell (Figure 2A). Untreated SM-BLM cells exhibited a higher number of RPA foci than untreated BLM+ cells (21.1 foci/cell vs. 3.7 foci/cell, respectively). HU induced RPA foci in both SM-BLM and BLM+ cells (105.8 foci/cell vs. 40.5 foci/cell, respectively; Figure 2B). The absolute increase was 84.7 foci/cell in SM-BLM cells compared to $36.8 \mathrm{foci} /$ cell in BLM+ cells. We noted that in HU-treated conditions in both SM-BLM and BLM+ cells, BLM co-localized to a high degree with a subset of RPA foci. These results ruled out the possibility that BLM SUMOylation is required for ssDNA accumulation; on the contrary, SM-BLM cells contained a vast excess of RPA foci in both untreated and HU-treated conditions. These data indicated that more ssDNA accumulated at stalled replication forks in SM-BLM compared to BLM+ cells.

\section{EXCESS CHROMATIN-BOUND RPA IN SM-BLM CELLS}

Because SM-BLM cells accumulated excess RPA foci, we predicted that more RPA would be bound to chromatin. To test this prediction, we isolated chromatin and nucleoplasmic fractions from BLM+ and SM-BLM cells untreated or treated with $5 \mathrm{mM}$ $\mathrm{HU}$ for $6 \mathrm{~h}$ and analyzed extracted proteins by immunoblot. In untreated conditions, the ratio of chromatin-bound RPA to total RPA was approximately equal in BLM+ and SM-BLM cells. After HU treatment, however, RPA shifted from nucleoplasmic fractions to chromatin-bound fractions in response to replication stress (Figure 3A). The ratio of chromatin-bound RPA to total RPA increased four fold in SM-BLM cells compared to two fold in BLM+ cells (Figure 3B). The increased amount of chromatinbound RPA after HU treatment in SM-BLM cells was consistent
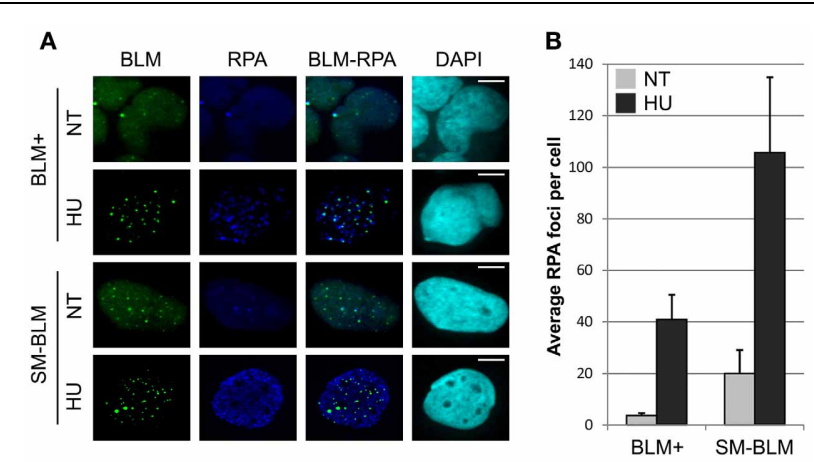

FIGURE 2 | Excess RPA accumulates at stalled replication forks in SUMO-mutant BLM cells. (A) Immunofluorescence images of BLM+ and SM-BLM cells untreated (NT) or treated with $0.5 \mathrm{mM} \mathrm{HU}$ for $24 \mathrm{~h}(\mathrm{HU})$ and stained for RPA. Bars indicate $10 \mu \mathrm{m}$. (B) Graphical representation of mean numbers of RPA foci. Three independent experiments were carried out for each of two BLM+ and two SM-BLM clones. Error bars represent the standard deviations of the results of each experiment. There were significantly more RPA foci in SM-BLM cells compared to BLM+ cells ( $p<0.001$ ) and HU treatment induced significantly more RPA foci in both cell lines $(p<0.001)$. Interaction was also observed indicating that the difference in effect of $\mathrm{HU}$ treatment on the two cell lines is significant $\left(p_{\text {int }}=0.002\right)$. $P$-values were calculated using mixed effects linear models as described in Materials and Methods. with the indirect immunofluorescence data that showed the accumulation of excess RPA foci. Together, these data indicated that BLM SUMOylation functions to limit the formation of excess RPA-ssDNA complex at stalled replication forks.

\section{DNA HELICASE ACTIVITY OF SUMOYLATED BLM IS NORMAL}

The excessive accumulation of fork-associated RPA raised the possibility that excess ssDNA was generated at stalled forks in SM-BLM cells due to a failure to suppress BLM's DNA unwinding activity at the fork. To test this hypothesis, we compared the DNA helicase activity of SUMOylated and unSUMOylated BLM in vitro. Purified His-tagged BLM helicase was SUMOylated in vitro using purified human E1, UBC9, and SUMO-2 in a reaction that required ATP. After incubation of the BLM with reaction components for $2 \mathrm{~h},>95 \%$ of the BLM was SUMOylated, and multiple moieties of SUMO-2 were attached to most of the BLM (Figure 4A). We then compared helicase activity of the SUMOylated and unSUMOylated BLM in two ways: (1) BLM SUMOylation reactions were prepared with or without ATP and reaction products were added directly to helicase assays; or, (2) SUMOylated and unSUMOylated BLM was partially purified from the SUMO reaction components by pull-down on nickel-NTA beads, after which bead-bound BLM was added to helicase assays. BLM activity was assayed on a ${ }^{32}$ P-labeled DNA replication-fork-like substrate (38-nucleotide duplex DNA with two 12-nucleotide ssDNA tails) and percent unwinding was measured by electrophoresis through nondenaturing polyacrylamide gels followed by autoradiography. In both types of experiment, the percent of DNA substrate unwound over time was indistinguishable for SUMOylated compared to unSUMOylated BLM (Figures 4B,C). These results showed that BLM SUMOylation does not function to suppress BLM's intrinsic DNA helicase activity. Therefore, the excessive accumulation of fork-associated RPA in SM-BLM cells was not the result of failure to suppress BLM's intrinsic DNA helicase activity.
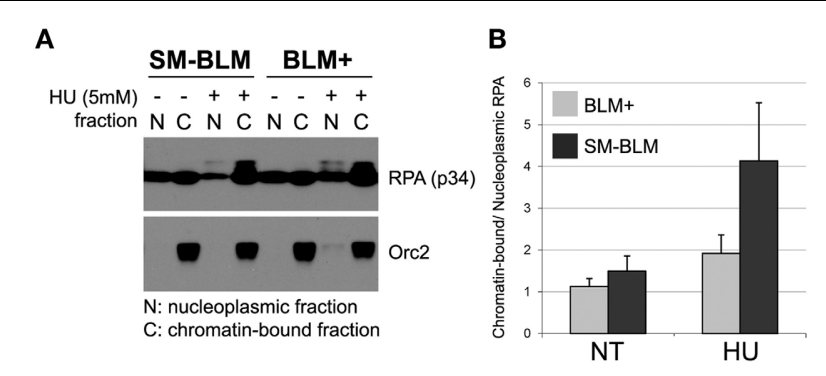

FIGURE 3 | Increased levels of RPA accumulate on chromatin in HU-treated SM-BLM cells. BLM+ and SM-BLM cells were untreated or treated with $5 \mathrm{mM}$ for $6 \mathrm{~h}$. (A) Chromatin and nucleoplasmic fractions were isolated from cells and solubilized RPA was analyzed by immunoblot using antibodies to the p34 subunit. Orc2 was used for quality control of the preparation of the chromatin-bound fraction. (B) Graph depicting the ratio of chromatin-bound RPA to nucleoplasmic RPA. A minimum of two independent experiments were carried out on each of two BLM+ and two SM-BLM clones. Error bars represent the standard deviations of the combined data. 
A

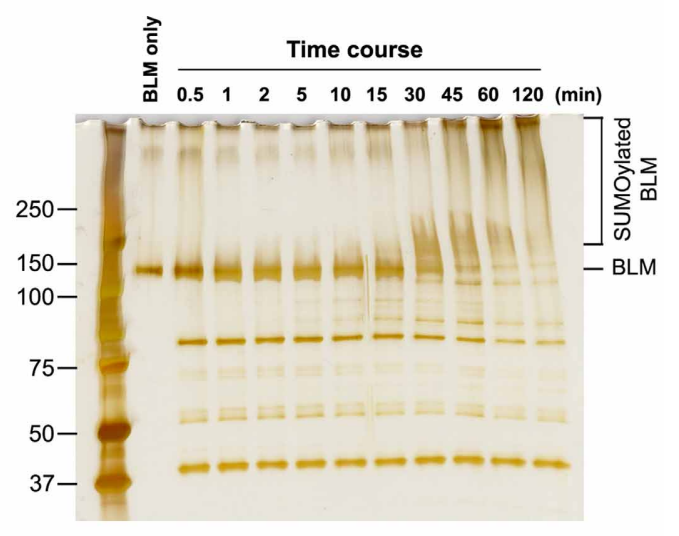

B

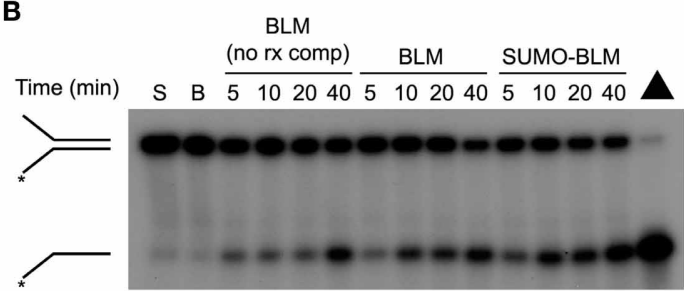

C

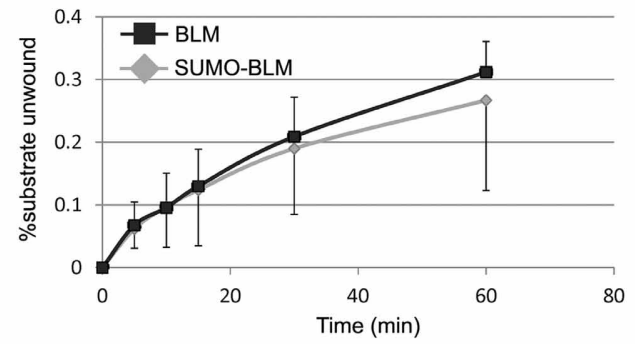

FIGURE 4 | DNA helicase activity of SUMOylated BLM is similar to unSUMOylated BLM. (A) BLM SUMOylation reactions were carried out, time points were removed, and the reactions were stopped with sample buffer. The reaction products were analyzed by sodium dodecyl sulphate-polyacrylamide gel electrophoresis followed by silver staining. (B) SUMOylated (SUMO-BLM) or unSUMOylated BLM (BLM) was prepared by incubating $200 \mathrm{ng}$ of purified recombinant BLM with SUMO reaction components in the presence (to make SUMOylated BLM) or absence (to make unSUMOylated BLM) of ATP. Helicase assays were performed by incubating the SUMO reaction products with ${ }^{32}$ P-labeled synthetic replication-fork substrate, and the helicase reactions were stopped at different times. The products were analyzed by non-denaturing polyacrylamide gel electrophoresis followed by autoradiography. The position of the forked duplex and the unwound product are indicated on the left. S, unreacted substrate; B, beads only with unreacted substrate; $\Delta$, boiled substrate; BLM (no rx comp), helicase reactions performed with BLM protein that was not exposed to SUMO reaction components as a positive control. (C) Graph showing quantification of the percentage of unwound substrate by SUMOylated and unSUMOylated BLM prepared in these experiments by nickel-NTA pull down. A minimum of two DNA unwinding experiments were performed for each method of BLM SUMOylation preparation shown in (B) and (C)

\section{EXCESS BLM-RPA COMPLEX FORMS IN SM-BLM CELLS}

BLM interacts with the $70 \mathrm{kD}$ subunit of RPA (RPA-70), and RPA stimulates BLM's DNA helicase activity (Brosh et al., 2000). If
BLM SUMOylation functions to inhibit BLM-RPA interaction, then excess RPA accumulation in SM-BLM cells could result from a failure to suppress BLM-RPA interaction. To test this hypothesis, we compared the binding of purified RPA to SUMOylated or unSUMOylated BLM in vitro. BLM SUMOylation reactions were prepared with or without ATP, reaction products were mixed with RPA, and BLM-RPA complexes were pulled down with nickel-NTA beads. The amount of BLM-bound RPA was then measured by immunoblot analysis with anti-RPA/p70 antibodies. In these assays, the amount of BLM-bound RPA-70 detected was the same whether or not BLM was SUMOylated (Figure 5A). These data suggested that BLM SUMOylation does not directly affect interaction between BLM and RPA.

Because BLM SUMOylation could affect BLM and RPA interaction indirectly, we compared BLM-RPA complex formation in vivo by immunoprecipitation of BLM from HU-treated and untreated BLM+ vs. SM-BLM cells. In untreated cells, little to no detectable RPA was pulled down with BLM in either cell line. However, in HU-treated cells, more RPA was pulled down with BLM in SM-BLM cells compared to BLM+ cells (Figure 5B). Finding increased BLM-RPA complex formation in SM-BLM cells correlated with the excessive accumulation of RPA foci in SM-BLM cells, and it suggested that BLM SUMOylation has a role in preventing the accumulation of excess ssDNA at stalled forks.

To test whether the increased BLM-RPA complex formation in SM-BLM cells was trivially a consequence of higher levels of RPA expression in these cells, we performed immunoblot analysis of untreated and HU-treated SM-BLM and BLM+ cells. Analysis of total cell extracts indicated that SM-BLM and BLM+ cells contained similar amounts of RPA protein with or without treatment with $\mathrm{HU}$ (Figure 5C). Together with the in vitro data, the results suggested that BLM SUMOylation affects BLM-RPA complex formation indirectly, possibly through the mediation of other proteins at the fork. We concluded that BLM SUMOylation regulates BLM-RPA interaction at stalled forks.

In these experiments, we noted that HU-induced RPA phosphorylation did not differ significantly between BLM+ and SMBLM cells, despite the presence of excess RPA at stalled forks in SM-BLM cells (Figure 5C).

\section{DISCUSSION}

The data presented here demonstrated that BLM SUMOylation regulates RPA and mediator accumulation at stalled replication forks, limiting the generation of excess ssDNA there. In HUtreated SM-BLM cells, a large excess of ssDNA accumulated at stalled forks, as evidenced by excess RPA foci and greater amounts of chromatin-bound RPA. With so much ssDNA generated at stalled forks in SM-BLM cells, we would expect large accumulations at the fork of both mediators BRCA2 and RAD52, and RAD51 as well. On the contrary, these proteins did not accumulate normally at stalled forks. These two observations are therefore evidence that BLM SUMOylation is necessary to license the HR mechanism at stalled forks, as mediator-dependent RAD51 complexes did not form on RPA-coated ssDNA in its absence. Because RAD51 loading onto ssDNA depends on mediator function, our results explain why RAD51 is not recruited to stalled 
A

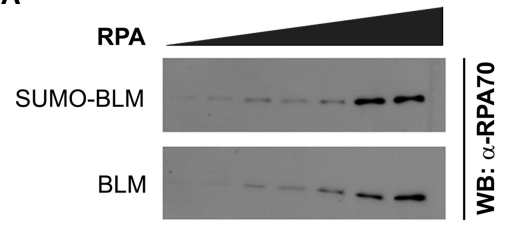

B
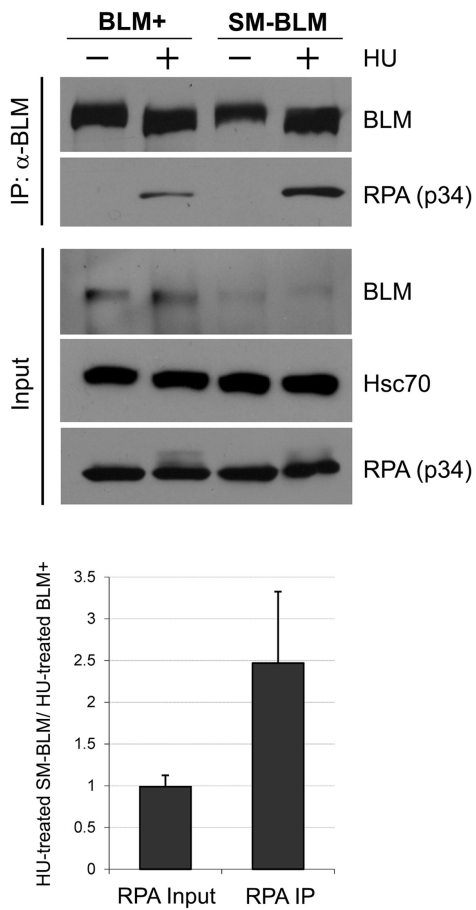

C

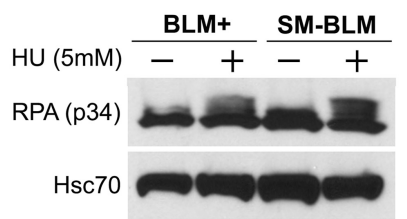

FIGURE 5 | Excess BLM-RPA complex forms in SM-BLM cells. (A)

SUMOylation of BLM did not affect BLM-RPA binding in vitro. SUMOylated (SUMO-BLM) or unSUMOylated (BLM) BLM were bound to Ni-NTA beads and incubated with increasing amounts of purified human RPA protein for $30 \mathrm{~min}$ at room temperature. The beads were washed three times. Bound RPA protein was analyzed by immunoblot with anti-RPA/p70 antibody. A bead-only experiment showed that no excess RPA bound to beads in the absence of BLM. (B) BLM binds more RPA in SM-BLM cells than BLM+ cells. Cells were untreated or treated with $5 \mathrm{mM} \mathrm{HU}$ for $6 \mathrm{~h}$.

Immunoprecipitation of RPA was carried out using antibodies to the GFP tag on GFP-BLM. Input consisted of $2 \%$ of the initial cell extracts. Hsc70 was used as a loading control. A minimum of two independent experiments were carried out for each of two BLM+ and two SM-BLM clones. The graph depicts the ratio of input and immunoprecipitated RPA in HU-treated SM-BLM cells over HU-treated BLM+ cells. (C) HU does not induce significantly different levels of RPA and phosphorylated RPA in SM-BLM cells compared to BLM+ cells. Cells were untreated or treated with $5 \mathrm{mM} \mathrm{HU}$ for $6 \mathrm{~h}$. Whole cell lysates were extracted and analyzed by immunoblot with antibodies to the p34 subunit of RPA. Hsc70 was used as a loading control. A minimum of two independent experiments were carried out for each of two BLM+ and of two SM-BLM clones. Error bars represent the standard deviations of the combined data. forks in SM-BLM cells; however, the mechanism explaining why mediators are not recruited is still enigmatic.

The uncoupling of DNA replication from the replicative helicase during fork stalling can lead to extended stretches of ssDNA and later to replication-associated DSBs that could be processed by exonucleases (Petermann and Helleday, 2010). Because RPAssDNA complexes form on both of these cellular substrates, we do not know a priori whether BLM SUMOylation is important before or after the replication fork breaks. In our previous work (Ouyang et al., 2009), we showed that 24-h HU treatment of SM-BLM cells did not lead to increased DSBs in comparison to untreated SM-BLM cells. Moreover, the effect of BLM SUMOylation on RAD51 accumulation at stalled forks was apparent after a 1-h HU treatment of SM-BLM cells before DSBs have had a chance to accumulate (Saintigny et al., 2001; Petermann et al., 2010). Therefore, these data suggest that BLM SUMOylation is important in the generation of ssDNA after helicase-polymerase uncoupling and before fork breakage.

Formally speaking, the function of BLM SUMOylation in HR licensing at the fork could result from either positive or negative regulatory effects or both. Previous work has shown that RPA interacts with BLM, inhibiting BLM's strand annealing activity while increasing the efficiency of its helicase activity (Brosh et al., 2000; Doherty et al., 2005; Bartos et al., 2006). SUMOylation did not inhibit BLM-RPA interaction in vitro, yet more BLMRPA complex formed in SM-BLM cells than in BLM+ cells after replication stress. Intriguingly, RPA is SUMOylated by SUMO2 after treatment with camptothecin (CPT), which generates replication-associated DSBs (Dou et al., 2010). Cells that express a SUMO-mutant RPA have defects in RAD51 accumulation and DSB repair at IR-induced DSBs and CPT-induced broken forks; but increased RPA SUMOylation was not reported to occur during replication stress with UV or $\mathrm{HU}$ treatment (Dou et al., 2010). Thus, SUMOylation of BLM and RPA seem to have similar effects on the accumulation of RAD51, one in the context of replication fork stalling and the other in the context of replication fork breakage. Further studies are needed to examine the role of SUMOylation on the interaction of RPA and BLM and the possible effects of SUMOylation on each protein's biochemical activities.

In normal cells, the amount of ssDNA that accumulates after replication stalling is regulated by damage sensing and checkpoint pathways that recruit proteins to stabilize the fork and inhibit further unwinding (Sleeth et al., 2007). Because BLM protein is recruited to the fork immediately after stalling occurs, it is possible that pathologically unrestricted SM-BLM protein generates excessive DNA unwinding at the fork. We showed that BLM helicase activity is not inhibited by SUMOylation; therefore, if SUMOylation prevents BLM from unwinding DNA at the fork, it must do so via interaction with another protein. Because RPA stimulates BLM helicase activity, it is possible that SUMOylation negates RPA's stimulatory effect. Alternatively, BLM SUMOylation could have an indirect effect on the accumulation of RPA-ssDNA complex through interaction with ATRmediated checkpoint signaling.

Another possibility is that BLM SUMOylation activates BLM or another protein's ssDNA annealing activity. A novel ssDNA 
annealing function was recently identified in the N-terminal portion of BLM distinct from an annealing activity reported for the helicase domain (Chen and Brill, 2010); this region coincides with the region containing the SUMO-acceptor sites and the region required for SUMO binding (Eladad et al., 2005; Zhu et al., 2008). This ssDNA annealing activity of BLM could function to stabilize stalled forks by reannealing excess ssDNA. The annealing helicase HARP (HepA-related protein) has a role in ssDNA annealing that leads to stabilization of stalled forks (Bansbach et al., 2009; Driscoll and Cimprich, 2009; Yuan et al., 2009), and BLM SUMOylation could de-repress or directly promote the activities of these proteins (Yusufzai and Kadonaga, 2008, 2010; Sen et al., 2012).

Many key components and functions of the HR machinery are highly conserved. An exception to this rule lies with mediator proteins. While Rad52 is an essential component in yeast HR (Pâques and Haber, 1999), RAD52 gene knockout in mice shows no significant effect on cell viability or on HR and DNA repair capacities (Rijkers et al., 1998; Yamaguchi-Iwai et al., 1998). Instead, the BRCA2 mediator protein, a component that is not present in budding yeast, plays a central role in mammalian DSB repair by HR (Xia et al., 2001; Yang et al., 2005; Carreira et al., 2009). Recent work has shown that RAD52 inactivation is synthetically lethal with BRCA2 deficiency in human cell lines, indicating overlapping functions between RAD52 and BRCA2 in RAD51-dependent HR repair (Feng et al., 2011). Some have suggested that RAD52's primary function is not in the repair of DSBs with two broken ends but in the protection and repair of stalled and broken replication forks (Wray et al., 2008; Feng et al., 2011). Our results showed that SM-BLM cells have a defect in the accumulation of both RAD52 and BRCA2 at stalled forks, indicating that BLM SUMOylation is necessary for efficient mediator recruitment to stalled replication forks. We note that RAD52 is SUMOylated by the MMS21 E3 ligase, which is part of the SMC5/6 complex, and RAD52's SUMOylation is required for its normal repair function (Sacher et al., 2006; Santa Maria et al., 2007; Torres-Rosell et al., 2007; Ohuchi et al., 2008; Altmannova et al., 2010). Recent work has indicated that mediators, RAD51, and the Fanconi anemia complex have functions in replication fork stabilization that are independent of their roles in DSB repair (Schlacher et al., 2011, 2012; Feng and Zhang, 2012). RAD52 has ssDNA annealing activity that could help stabilize the replication fork (Wu et al., 2008; Grimme et al., 2010); consequently, the failure to recruit mediators to stalled forks in SM-BLM cells could explain the accumulation of excess RPA-ssDNA, if mediators are required for ssDNA annealing at the fork, or it could be an independent consequence of unSUMOylated BLM activity at the stalled fork.

While excess accumulation of RPA in HU-treated SM-BLM cells could result from either excessive DNA unwinding or impaired ssDNA re-annealing, there is yet another possible explanation relating to replication dynamics. Work in yeast and mammalian cells has shown that replication forks collapsed by prolonged replication stalling do not restart, and replication is rescued instead by new origin firing (Davies et al., 2007; Petermann et al., 2010). In SM-BLM cells, stalled replication forks may collapse more readily, leading to initiation of new forks to compensate for the loss. In this model, the excess RPA foci would be accounted for by activation of dormant replication origins.

The SUMO pathway prevents aberrant recombination events from occurring at damaged replication forks. It positively regulates DSB repair by HR (Liberi et al., 2005; Branzei et al., 2006; Burgess et al., 2007) and it can influence repair pathway choice (Ulrich, 2009; Yang et al., 2011). Multiple components of the HR pathway are SUMOylated, but in most cases a complete mechanistic understanding of SUMO's role in functional regulation of its substrates is lacking. Protein-protein interactions between SUMO binding sites and SUMOs could be important in the recruitment of DNA repair factors such as 53BP1 and BRCA1 to the repair site (Bergink and Jentsch, 2009; Galanty et al., 2009; Morris et al., 2009). Recent work on the SUMOtargeted ubiquitin ligase RNF4 has suggested that SUMOylation could be important for dissociation of proteins such as MDC1 from the repair site (Galanty et al., 2012; Luo et al., 2012; Yin et al., 2012; Vyas et al., 2013), but ubiquitylation by RNF4 could also serve to recruit proteins with ubiquitin-binding sites by making hybrid SUMO-ubiquitin chains (Guzzo et al., 2012). From all these studies, SUMO seems to govern important turnover transitions, promulgating cycles of association and dissociation at the repair site: BLM SUMOylation could both assist in the recruitment of factors like RAD52 and RAD51 and it could also remove BLM from the repair site; both of these roles for SUMOylation would be absent in the SM-BLM protein.

BLM has many functions throughout the processes of DNA replication and $\mathrm{HR}$ repair. How these functions are regulated is an important question. SUMOylation is a dynamic process that confers diverse and unique roles to its substrate proteins. The present work shed light on how BLM SUMOylation regulates RPA and mediator functions at stalled forks, and more broadly, how replication forks are maintained under stress when HR is called into play. Our evidence suggests that BLM SUMOylation functions as a licensing mechanism that regulates and permits execution of the HR mechanism at damaged replication forks. The next step is to dissect the specific recruitment and turnover functions of SUMOylation on BLM and other proteins in this licensing mechanism.

\section{MATERIALS AND METHODS ANTIBODIES}

For BLM immunoblot analysis, rabbit polyclonal anti-BLM antibodies raised against the first 431 amino acids of human BLM (Beresten et al., 1999) were used. For other immunoblot analyses, rabbit polyclonal anti- $\gamma-\mathrm{H} 2 \mathrm{AX}$ antibodies (Abcam), mouse monoclonal anti-RPA/p34 antibody (Neomarkers), mouse monoclonal anti-RPA/p70 antibody (Neomarkers), rat monoclonal anti-ORC2 antibody (Abcam), and rat monoclonal anti-Hsc70 antibody (Assay Design) were used. Horseradish peroxidase-linked anti-mouse, anti-rabbit (Amersham), and anti-rat (Jackson ImmunoResearch) antibodies were used as secondary antibodies. Agarose conjugated rat monoclonal antiGFP antibody D153-8 (MBL) was used for immunoprecipitation experiments. For indirect immunofluorescence, we used mouse monoclonal anti- $\gamma-\mathrm{H} 2 \mathrm{AX}$ antibody (Upstate), rabbit polyclonal anti-RAD51 antibodies PC130 (Calbiochem), rabbit polyclonal 
anti-RAD52 antibodies sc-8250 (Santa Cruz Biotechnology), mouse anti-BRCA2 antibodies 05-666 (Millipore), mouse monoclonal anti-RPA/p34 antibody (Neomarkers), Cy-5-labeled donkey anti-rabbit antibodies (Jackson ImmunoResearch), Alexa Fluor 594-labeled goat anti-mouse, Alexa Fluor 594-labeled goat anti-rabbit, and Alexa Fluor 647-labeled goat anti-mouse antibodies (Invitrogen).

\section{IMMUNOFLUORESCENCE AND IMAGE ANALYSIS}

The generation and characterization of BLM+ and SM-BLM cells was described previously (Eladad et al., 2005; Ouyang et al., 2009). Briefly, the SV40-transformed BS fibroblast cell line GM08505 was transfected with normal GFP-BLM or the SM-GFP BLM expression constructs, clones were isolated and selected for analysis that expressed similar amounts of GFP-BLM proteins. For indirect immunofluorescence, BLM+ and SM-BLM cells were seeded on coverslips and then treated with $0.5 \mathrm{mM} \mathrm{HU}$ in culture medium for $24 \mathrm{~h}$. At the end of HU treatment, cells were washed and fixed and then stained with anti-RAD51, - BRCA2, - RAD52, or -RPA antibodies, and counterstained with appropriate secondary antibodies labeled with Alexa Fluor (Invitrogen). Fixation and staining was performed as described previously (Eladad et al., 2005; Ouyang et al., 2009). Coverslips were mounted with Prolong Gold antifade reagent containing 4',6-diamidino2-phenylindole (DAPI; Invitrogen). Images were captured on a spinning disk confocal microscope (Carl Zeiss, LSM-510), and data were collected using Slidebook 4.1 software. Z-stacks were captured using a $100 \times$ oil immersion objective and the optical slice thickness was $0.2 \mu \mathrm{m}$. The BRCA2 data was collected on a laser-scanning confocal microscope (Carl Zeiss, LSM-710) and a single plane was imaged.

A focus was defined as a defined area of the nucleus greater than the minimum area of optical resolution $\left(>0.125 \mu \mathrm{m}^{2}\right)$ in at least one Z-stack in which the fluorescence intensity was greater than the background fluorescence intensity of the nucleoplasm. The maximum number of foci that could be counted in these cells was 150. A typical immunofluorescence experiment consisted of assessment of 30-50 cells per condition. The data presented are from two to three independent experiments performed on two to three clones of each type. Immunofluorescence images for figures were created using Image J and Metamorph software (Molecular Devices).

\section{IMMUNOBLOT ANALYSES}

Proteins from cell lysates, chromatin, and nucleoplasmic fractions, and immunoprecipitates were separated on 4-15\% sodium dodecyl sulphate (SDS)/polyacrylamide gradient gels (Bio-Rad) and transferred on to polyvinylidene fluoride membranes (Bio$\mathrm{Rad})$. The membranes were blocked for $1 \mathrm{~h}$ at room temperature with Tris-buffered saline (Boston Bioproducts) containing 0.1\% Tween 20 and 5\% powdered milk (Bio-Rad), washed, and subsequently probed with appropriate primary antibodies either for $2 \mathrm{~h}$ at room temperature or overnight at $4^{\circ} \mathrm{C}$. The membranes were then incubated with the appropriate horseradish peroxidase-linked secondary antibodies for $1 \mathrm{~h}$ at room temperature, washed, and incubated with Western Lightning-ECL, Enhanced Chemiluminescence reagent (Perkin Elmer) for $5 \mathrm{~min}$ at room temperature. Proteins labeled with antibodies on the membrane were visualized by detection on film.

\section{CHROMATIN ISOLATION}

Chromatin preparations were made by the method of Méndez and Stillman (2000). Briefly, cells treated with or without $5 \mathrm{mM}$ $\mathrm{HU}$ for $6 \mathrm{~h}$ were harvested by centrifugation, washed in PBS, and resuspended in buffer A (10 mM HEPES, pH 7.9, $10 \mathrm{mM}$ $\mathrm{KCl}, 1.5 \mathrm{mM} \mathrm{MgCl} 2,0.34 \mathrm{M}$ sucrose, $10 \%$ glycerol, $1 \mathrm{mM}$ DTT, $0.1 \mathrm{mM}$ PMSF, and protease inhibitors cocktail). Triton X-100 $(0.1 \%)$ was added, and the cells were incubated for $5 \mathrm{~min}$ on ice. Nuclei were collected in the pellet by low-speed centrifugation at $1300 \times \mathrm{g}$ for $4 \mathrm{~min}$ at $4^{\circ} \mathrm{C}$. Nuclei were washed once in buffer A, and then lysed in buffer B ( $3 \mathrm{mM}$ EDTA, $0.2 \mathrm{mM}$ EGTA, $1 \mathrm{mM}$ DTT, protease inhibitors cocktail). Nucleoplasmic proteins were separated from chromatin-bound proteins by centrifugation at $1700 \times \mathrm{g}$ for $5 \mathrm{~min}$ at $4^{\circ} \mathrm{C}$. Nucleoplasmic fractions were collected in the supernatant. The chromatin pellet was washed once in buffer B, and centrifuged again under the same conditions. The final chromatin pellet was resuspended in Laemmli sample buffer. HU treatment was performed at $5 \mathrm{mM}$ for $6 \mathrm{~h}$ so that BLM quantities would be nearly the same in untreated and treated cells. With respect to the phenotypes studied here, there are no major differences between the 6- and 24-h treatments (Ouyang et al., 2009).

\section{IMMUNOPRECIPITATION}

Cells treated with or without $5 \mathrm{mM} \mathrm{HU}$ for $6 \mathrm{~h}$ were harvested, washed twice in PBS buffer, and resuspended in NP-40 lysis buffer (Boston Bioproducts) containing protease inhibitors (Roche) for $30 \mathrm{~min}$. Cell lysates were cleared by centrifugation (15 min, $14,000 \times \mathrm{g}, 4^{\circ} \mathrm{C}$ ). Cleared cell lysates were then incubated with $20 \mu \mathrm{l}$ of agarose beads conjugated with anti-GFP antibodies and allowed to mix overnight at $4^{\circ} \mathrm{C}$. Proteins bound to beads were separated from the supernatant by centrifugation ( $3 \mathrm{~min}, 2000 \times$ $\mathrm{g}, 4^{\circ} \mathrm{C}$ ) and washed with 5 times volume of lysis buffer. Proteins were extracted from the beads by boiling in Laemmli buffer (Boston Bioproducts) for $5 \mathrm{~min}$ and subsequently analyzed by immunoblot.

\section{SUMOYLATION OF BLM}

Two hundred nanograms of His-tagged full-length BLM, purified according to the method of Karow et al. (1997), was SUMOylated in a $50 \mu \mathrm{l}$ reaction containing $10 \mathrm{mM}$ HEPES ( $\mathrm{pH} 7.3$ ), $110 \mathrm{mM}$ potassium acetate, $2 \mathrm{mM} \mathrm{MgCl}, 2 \mathrm{mM}$ ATP, and purified recombinant proteins (Zhu et al., 2008), including $200 \mathrm{ng} \mathrm{E1}, 100 \mathrm{ng}$ UBC9, and $200 \mathrm{ng}$ SUMO-2, at $37^{\circ} \mathrm{C}$ for increasing amounts of time $(0.5,1,2,5,10,15,30,45,60$, and $120 \mathrm{~min})$. Reactions were stopped by the addition of sample buffer and subsequently analyzed by SDS-polyarcylamide gel electrophoresis followed by Silver staining (Pierce Silver Stain Kit, Thermo Scientific) per the manufacturer's instructions.

\section{DNA SUBSTRATE}

Oligonucleotides for the fork substrate designed according to Mohaghegh et al. (2001) were purchased from Integrated DNA Technologies. A single oligonucleotide was $5^{\prime}$-end-labeled with $\left[\gamma-{ }^{32} \mathrm{P}\right]$ ATP (PerkinElmer Life Sciences) using T4 polynucleotide kinase in $\mathrm{PNK}$ buffer at $37^{\circ} \mathrm{C}$ for $30 \mathrm{~min}$ per the manufacturer's instructions (New England Biolabs). The labeled substrate was purified using QIAquick Nucleotide Removal kit (Qiagen). The $\left[\gamma^{32} \mathrm{P}\right]$ ATP-labeled oligonucleotide was then annealed 
with a two fold excess of the unlabeled complementary oligonucleotide in annealing buffer $(40 \mathrm{mM}$ Tris- $\mathrm{HCl}, \mathrm{pH} 8.0,50 \mathrm{mM}$ $\mathrm{NaCl}$ ) by heating at $95^{\circ} \mathrm{C}$ for $5 \mathrm{~min}$ and then cooling slowly to room temperature.

\section{HELICASE ASSAY}

Equal amounts of SUMOylated BLM and unSUMOylated BLM, respectively, were added to $100 \mu \mathrm{l}$ of nickel-NTA bead slurry (Qiagen) that had been blocked in $0.5 \%$ BSA for $2 \mathrm{~h}$ at room temperature and then washed two times in BLM binding buffer (60 mM Tris- $\mathrm{HCl}, \mathrm{pH} 7.4,20 \mathrm{mM} \mathrm{KCl}$ ). BLM was bound to beads for $1 \mathrm{~h}$ at room temperature and washed two times in BLM binding buffer. Helicase reactions were performed with $20 \mathrm{ng}$ BLM in $10 \mathrm{mM}$ Tris- $\mathrm{HCl}$ ( $\mathrm{pH} 7.5$ ), $4 \mathrm{mM} \mathrm{MgCl} 2,1 \mathrm{mM}$ dithiothreitol, $2 \mathrm{mM}$ ATP, $0.1 \mathrm{mg} / \mathrm{ml} \mathrm{BSA}$, and $0.3 \mathrm{nM}$ of labeled helicase substrate at $37^{\circ} \mathrm{C}$ for either $5,10,15,20,30,40$, or $60 \mathrm{~min}$. Helicase reactions were stopped by adding gel loading dye $(50 \mathrm{mM}$ EDTA, 40\% glycerol, 0.9\% SDS, 0.05\% bromophenol blue, and $0.05 \%$ xylene cyanol). Reaction products were analyzed by electrophoresis through pre-cast $10 \%$ non-denaturing polyacrylamide gels (Bio-Rad). The amount of substrate unwound was measured by autoradiography using a phoshorimager (Molecular Dynamics).

\section{In vitro BINDING ASSAY}

Two hundred nanograms of SUMOylated and unSUMOylated BLM, respectively, were added to $200 \mu \mathrm{l}$ of Ni-NTA bead slurry (Qiagen) that had been previously incubated in Blocking Buffer (PBS, 0.5\% Tween 20, 3\% BSA) for $2 \mathrm{~h}$ at room temperature and subsequently washed three times with PBS. Increasing concentrations of purified RPA were then incubated with $20 \mathrm{ng}$ of either SUMOylated BLM or unSUMOylated BLM bound to nickel-NTA beads, respectively, in binding Buffer (50 mM Tris- $\mathrm{HCl}, \mathrm{pH} 7.4,5 \mathrm{mM} \mathrm{MgCl}_{2}, 5 \mathrm{mM}$ ATP, $100 \mu \mathrm{g} / \mathrm{ml} \mathrm{BSA}$, and $50 \mathrm{mM} \mathrm{NaCl}$ ) for $30 \mathrm{~min}$ at room temperature. Beads were then washed three times in binding buffer. The amount of RPA-70 protein bound to SUMOylated BLM

\section{REFERENCES}

Altmannova, V., Eckert-Boulet, N., Arneric, M., Kolesar, P., Chaloupkova, R., Damborsky, J., et al. (2010). Rad52 SUMOylation affects the efficiency of the DNA repair. Nucleic Acids Res. 38, 4708-4721. doi: 10.1093/nar/gkq195

Balajee, A. S., and Geard, C. R. (2004). Replication protein A and gamma-H2AX foci assembly is triggered by cellular response to DNA double-strand breaks. Exp. Cell Res. 300, 320-334. doi: 10.1016/j.yexcr. 2004.07.022

Bansbach, C. E., Bétous, R., Lovejoy, C. A., Glick, G. G., and Cortez, D. (2009). The annealing helicase SMARCAL1 maintains genome integrity at stalled replication forks.

or unSUMOylated BLM was analyzed by immunoblot with anti-RPA/p70 antibodies.

\section{STATISTICAL ANALYSIS}

Because observations within each clone may be correlated, we used mixed effects linear models to test the data for statistical significance. In the mixed effects models, each clone was treated as a random effect and the experimental variables were treated as fixed effects. For testing changes in the number of foci/cell, cell type (BLM or SM), treatment (with and without HU), and interaction terms for cell type by treatment were treated as fixed effects. Because the foci data were not normally distributed, we used a generalized estimating equation approach that can account for non-normal correlated data with non-homogeneous variances.

\section{ACKNOWLEDGMENTS}

The authors thank Jianmei Zhu for providing purified proteins for carrying out SUMOylation reactions, Vytas Bindokas in the University of Chicago Integrated Microscopy Core Facility, and $\mathrm{Ke} \mathrm{Ma}$ in the University of Illinois Confocal Microscopy Facility for assistance with the immunofluorescence. We also thank Hui Xie in the statistics core in the UIC Center for Clinical and Translational Science (CCTS), which is supported under award UL1RR029879 from the National Center for Research Resources, for his help with the statistical analysis. Research reported in this publication was supported by the National Cancer Institute of the National Institutes of Health under award numbers R01CA140804 and U01CA153060. The content is solely the responsibility of the authors and does not necessarily represent the official views of the National Institutes of Health.

\section{SUPPLEMENTARY MATERIAL}

The Supplementary Material for this article can be found online at: frontiersin.org/Cancer_Genetics/10.3389/ fgene.2013.00167/abstract

N431 as oligomeric complexes. Protein Expr. Purif. 17, 239-248. doi: 10.1006/prep.1999.1135

Bergink, S., and Jentsch, S. (2009). Principles of ubiquitin and SUMO modifications in DNA repair. Nature 458, 461-467. doi: 10.1038/nature07963

Branzei, D., Sollier, J., Liberi, G., Zhao, X., Maeda, D., Seki, M., et al. (2006). Ubc9- and mms21mediated sumoylation counteracts recombinogenic events at damaged replication forks. Cell 127, 509-522. doi: 10.1016/j.cell. 2006.08.050

Brosh, R. M. Jr., Li, J. L., Kenny, M. K., Karow, J. K., Cooper, M. P., Kureekattil, R. P., et al. (2000). Replication protein A physically interacts with the Bloom's syndrome protein and stimulates its helicase activity. J. Biol. Chem. 275, 23500-23508. doi: 10.1074/jbc.M001557200

Burgess, R. C., Rahman, S., Lisby, M., Rothstein, R., and Zhao, X. (2007). The Slx5-Slx8 complex affects sumoylation of DNA repair proteins and negatively regulates recombination. Mol. Cell. Biol. 27, 6153-6162. doi: 10.1128/MCB.00787-07

Bussen, W., Raynard, S., Busygina, V., Singh, A. K., and Sung, P. (2007). Holliday junction processing activity of the BLM-Topo IIIalpha-BLAP75 complex. J. Biol. Chem. 282, 31484-31492. doi: 10.1074/jbc.M706116200

Byun, T. S., Pacek, M., Yee, M. C., Walter, J. C., and Cimprich, K. A. (2005). Functional uncoupling 
of MCM helicase and DNA polymerase activities activates the ATR-dependent checkpoint. Genes Dev. 19, 1040-1052. doi: 10.1101/gad.1301205

Carreira, A., Hilario, J., Amitani, I., Baskin, R. J., Shivji, M. K., Venkitaraman, A. R., et al. (2009). The BRC repeats of BRCA2 modulate the DNA-binding selectivity of RAD51. Cell 136, 1032-1043. doi: 10.1016/j.cell.2009. 02.019

Chaganti, R. S., Schonberg, S., and German, J. (1974). A manyfold increase in sister chromatid exchanges in Bloom's syndrome lymphocytes. Proc. Natl. Acad. Sci. U.S.A. 71, 4508-4512. doi: 10.1073/pnas.71.11.4508

Chan, K. L., North, P. S., and Hickson, I. D. (2007). BLM is required for faithful chromosome segregation and its localization defines a class of ultrafine anaphase bridges. EMBO J. 26, 3397-3409. doi: 10.1038/sj.emboj.7601777

Chan, K. L., Palmai-Pallag, T., Ying, S., and Hickson, I. D. (2009). Replication stress induces sisterchromatid bridging at fragile site loci in mitosis. Nat. Cell Biol. 11, 753-760. doi: 10.1038/ncb1882

Chen, C. F., and Brill, S. J. (2010). An essential DNA strand-exchange activity is conserved in the divergent N-termini of BLM orthologs. ЕMBO J. 29, 1713-1725. doi: 10.1038/emboj.2010.61

Davalos, A. R., and Campisi, J. (2003). Bloom syndrome cells undergo p53-dependent apoptosis and delayed assembly of BRCA1 and NBS1 repair complexes at stalled replication forks. J. Cell Biol. 162, 1197-1209. doi: 10.1083/jcb.200304016

Davies, S. L., North, P. S., Dart, A., Lakin, N. D., and Hickson, I. D. (2004). Phosphorylation of the Bloom's syndrome helicase and its role in recovery from Sphase arrest. Mol. Cell. Biol. 24, 1279-1291. doi: 10.1128/MCB.24.3. 1279-1291.2004

Davies, S. L., North, P. S., and Hickson, I. D. (2007). Role for BLM in replication-fork restart and suppression of origin firing after replicative stress. Nat. Struct. Mol. Biol. 14, 677-679. doi: 10.1038/nsmb1267

Doherty, K. M., Sommers, J. A., Gray, M. D., Lee, J. W., von Kobbe, C., Thoma, N. H., et al. (2005). Physical and functional mapping of the replication protein a interaction domain of the werner and bloom syndrome helicases. J. Biol.
Chem. 280, 29494-29505. doi: 10.1074/jbc.M500653200

Dou, H., Huang, C., Singh, M., Carpenter, P. B., and Yeh, E. T. (2010). Regulation of DNA repair through deSUMOylation and SUMOylation of replication protein A complex. Mol. Cell 39, 333-345. doi: 10.1016/j.molcel.2010.07.021

Driscoll, R., and Cimprich, K. A. (2009). HARPing on about the DNA damage response during replication. Genes Dev. 23 , 2359-2365. doi: 10.1101/ gad. 1860609

Eladad, S., Ye, T.-Z., Hu, P., Leversha, M., Beresten, S., Matunis, M. J., et al. (2005). Intra-nuclear trafficking of the BLM helicase to DNA damage-induced foci is regulated by SUMO modification. Hum. Mol. Genet. 14, 1351-1365. doi: 10.1093/hmg/ddi145

Ellis, N. A., Groden, J., Ye, T. Z., Straughen, J., Lennon, D. J., Ciocci, S., et al. (1995). The Bloom's syndrome gene product is homologous to RecQ helicases. Cell 83, 655-666. doi: 10.1016/0092-8674(95)90105-1

Feng, Z., Scott, S. P., Bussen, W., Sharma, G. G., Guo, G., Pandita, T. K., et al. (2011). Rad52 inactivation is synthetically lethal with BRCA2 deficiency. Proc. Natl. Acad. Sci. U.S.A. 108, 686-691. doi: 10.1073/pnas.1010959107

Feng, Z., and Zhang, J. (2012). A dual role of BRCA1 in two distinct homologous recombination mediated repair in response to replication arrest. Nucleic Acids Res. 40, 726-738. doi: 10.1093/nar/gkr748

Galanty, Y., Belotserkovskaya, R., Coates, J., Polo, S., Miller, K. M., and Jackson, S. P. (2009). Mammalian SUMO E3-ligases PIAS1 and PIAS4 promote responses to DNA double-strand breaks. Nature 462, 935-939. doi: 10.1038/ nature08657

Galanty, Y., Belotserkovskaya, R., Coates, J., and Jackson, S. P. (2012). RNF4, a SUMO-targeted ubiquitin E3 ligase, promotes DNA double-strand break repair. Genes Dev. 26, 1179-1195. doi: 10.1101/gad.188284.112

German, J. (1964). Cytological evidence for crossing-over in vitro in lymphoid cells. Science 144, 298-301. doi: 10.1126/science.144. 3616.298

German, J., and Crippa, L. P. (1966). Chromosomal breakage in diploid cell lines from Bloom's syndrome and Fanconi's anemia. Ann. Genet. 9, 143-154.

Grimme, J. M., Honda, M., Wright, R., Okuno, Y., Rothenberg, E., Mazin,
A. V., et al. (2010). Human Rad52 binds and wraps single-stranded DNA and mediates annealing via two hRad52-ssDNA complexes. Nucleic Acids Res. 38, 2917-2930. doi: 10.1093/nar/gkp1249

Groden, J., Nakamura, Y., and German, J. (1990). Molecular evidence that homologous recombination occurs in proliferating human somatic cells. Proc. Natl. Acad. Sci. U.S.A. 87, 4315-4319. doi: 10.1073/pnas.87.11.4315

Guzzo, C. M., Berndsen, C. E., Zhu, J., Gupta, V., Datta, A. Greenberg, R. A., et al. (2012). RNF4-dependent hybrid SUMOubiquitin chains are signals for RAP80 and thereby mediate the recruitment of BRCA1 to sites of DNA damage. Sci. Signal. 5, ra88. doi: 10.1126/scisignal.2003485

Hand, R., and German, J. (1975). A retarded rate of DNA chain growth in Bloom's syndrome. Proc. Natl. Acad. Sci. U.S.A. 72, 758-762. doi 10.1073/pnas.72.2.758

Heyer, W. D., Ehmsen, K. T., and Liu, J. (2010). Regulation of homologous recombination in eukaryotes. Annu. Rev. Genet. 44, 113-139. doi: 10.1146/annurevgenet-051710-150955

Karow, J. K., Chakraverty, R. K., and Hickson, I. D. (1997). The Bloom's syndrome gene product is a $3^{\prime}-5$ ' DNA helicase. J. Biol. Chem. 272, 30611-30614. doi: 10.1074/jbc.272.49.30611

Lambert, S., Mizuno, K., Blaisonneau, J., Martineau, S., Chanet, R., Fréon, K., et al. (2010). Homologous recombination restarts blocked replication forks at the expense of genome rearrangements by template exchange. Mol. Cell 39, 346-359. doi: 10.1016/j.molcel.2010.07.015

Langlois, R. G., Bigbee, W. L., Jensen, R. H., and German, J. (1989). Evidence for increased in vivo mutation and somatic recombination in Bloom's syndrome. Proc. Natl. Acad. Sci. U.S.A. 86, 670-674. doi 10.1073/pnas.86.2.670

LaRocque, J. R., Stark, J. M., Oh, J., Bojilova, E., Yusa, K., Horie, K., et al. (2011). Interhomolog recombination and loss of heterozygosity in wild-type and Bloom syndrome helicase (BLM)-deficient mammalian cells. Proc. Natl. Acad. Sci. U.S.A. 108, 11971-11976. doi 10.1073/pnas.1104421108

Li, W., Kim, S. M., Lee, J., and Dunphy, W. G. (2004). Absence of BLM leads to accumulation of chromosomal DNA breaks during both unperturbed and disrupted $\mathrm{S}$ phases. J. Cell Biol. 165, 801-812. doi: $10.1083 /$ jcb. 200402095

Liberi, G., Maffioletti, G., Lucca, C., Chiolo, I., Baryshnikova, A., Cotta-Ramusino, C., et al. (2005). Rad51-dependent DNA structures accumulate at damaged replication forks in sgs1 mutants defective in the yeast ortholog of BLM RecQ helicase. Genes Dev. 19, 339-350. doi: 10.1101/ gad. 322605

Lönn, U., Lönn, S., Nylen, U., Winblad, G., and German, J. (1990). An abnormal profile of DNA replication intermediates in Bloom's syndrome. Cancer Res. 50, 3141-3145.

Lukas, C., Savic, V., Bekker-Jensen, S., Doil, C., Neumann, B., Pedersen, R. S., et al. (2011). 53BP1 nuclear bodies form around DNA lesions generated by mitotic transmission of chromosomes under replication stress. Nat. Cell Biol. 13, 243-253. doi: $10.1038 /$ ncb2201

Luo, K., Zhang, H., Wang, L., Yuan, J., and Lou, Z. (2012). Sumoylation of $\mathrm{MDC1}$ is important for proper DNA damage response. EMBO J. 31, 3008-3019. doi: 10.1038/emboj.2012.158

Mimitou, E. P., and Symington, L. S. (2009). Nucleases and helicases take center stage in homologous recombination. Trends Biochem. Sci. 34, 264-272. doi: 10.1016/j.tibs.2009.01.010

Méndez, J., and Stillman, B. (2000). Chromatin association of human origin recognition complex, cdc6, and minichromosome maintenance proteins during the cell cycle: assembly of prereplication complexes in late mitosis. Mol. Cell. Biol. 20, 8602-8612. doi: $\quad 10.1128 / \mathrm{MCB} .20 .22 .8602-$ 8612.2000

Mohaghegh, P., Karow, J. K., Brosh, R. M. Jr., Bohr, V. A., and Hickson, I. D. (2001). The Bloom's and Werner's syndrome proteins are DNA structure-specific helicases. Nucleic Acids Res. 29, 2843-2849. doi: 10.1093/nar/29.13.2843

Morris, J. R., Boutell, C., Keppler, M., Densham, R., Weekes, D. Alamshah, A., et al. (2009). The SUMO modification pathway is involved in the BRCA1 response to genotoxic stress. Nature 462, 886-890. doi: 10.1038/nature08593 Nimonkar, A. V., Ozsoy, A. Z., Genschel, J., Modrich, P., and Kowalczykowski, S. C. (2008). Human exonuclease 1 and BLM helicase interact to resect DNA and initiate DNA repair. Proc. Natl. Acad. Sci. U.S.A. 105, 
16906-16911. doi: 10.1073/pnas. 0809380105

Nimonkar, A. V., Genschel, J., Kinoshita, E., Polaczek, P., Campbell, J. L., Wyman, C., et al. (2011). BLM-DNA2-RPAMRN and EXO1-BLM-RPA-MRN constitute two DNA end resection machineries for human DNA break repair. Genes Dev. 25, 350-362. doi: 10.1101/gad.2003811

Ohuchi, T., Seki, M., Branzei, D., Maeda, D., Ui, A., Ogiwara, H., et al. (2008). Rad52 sumoylation and its involvement in the efficient induction of homologous recombination. DNA Repair 7, 879-889. doi: 10.1016/j.dnarep.2008.02.005

Ouyang, K. J., Woo, L. L., Zhu, J., Huo, D., Matunis, M. J., and Ellis, N. A. (2009). SUMO modification regulates BLM and RAD51 interaction at damaged replication forks. PLoS Biol. 7:e1000252. doi: 10.1371/journal.pbio. 1000252

Pâques, F., and Haber, J. E. (1999). Multiple pathways of recombination induced by double-strand breaks in Saccharomyces cerevisiae. Microbiol. Mol. Biol. Rev. 63, 349-404.

Petermann, E., and Helleday, T. (2010). Pathways of mammalian replication fork restart. Nat. Rev. Mol. Cell Biol. 11, 683-687. doi: 10.1038/nrm2974

Petermann, E., Orta, M. L., Issaeva, N., Schultz, N., and Helleday, T. (2010). Hydroxyurea-stalled replication forks become progressively inactivated and require two different RAD51-mediated pathways for restart and repair. Mol. Cell 37, 492-502. doi: 10.1016/j.molcel.2010.01.021

Rao, V. A., Conti, C., GuirouilhBarbat, J., Nakamura, A., Miao, Z. H., Davies, S. L., et al. (2007). Endogenous gamma-H2AXATM-Chk2 checkpoint activation in Bloom's syndrome helicase deficient cells is related to DNA replication arrested forks. Mol. Cancer Res. 5, 713-724. doi: 10.1158/1541-7786.MCR-07-0028

Raynard, S., Bussen, W., and Sung, P. (2006). A double Holliday junction dissolvasome comprising BLM, topoisomerase IIIalpha, and BLAP75. J. Biol. Chem. 281, 13861-13864. doi: 10.1074/jbc.C600051200

Rijkers, T., Van Den Ouweland, J., Morolli, B., Rolink, A. G., Baarends, W. M., Van Sloun, P. P., et al. (1998). Targeted inactivation of mouse RAD52 reduces homologous recombination but not resistance to ionizing radiation. Mol. Cell. Biol. 18, 6423-6429.
Sacher, M., Pfander, B., Hoege, C., and Jentsch, S. (2006). Control of $\operatorname{Rad} 52$ recombination activity by double-strand break-induced SUMO modification. Nat. Cell Biol. 8, 1284-1290. doi: 10.1038/ncb1488 Saintigny, Y., Delacôte, F., Varès, G., Petitot, F., Lambert, S., Averbeck, D., et al. (2001). Characterization of homologous recombination induced by replication inhibition in mammalian cells. EMBO J. 20, 3861-3870. doi: 10.1093/emboj/20.14.3861

Santa Maria, S. R., Gangavarapu, V., Johnson, R. E., Prakash, L., and Prakash, S. (2007). Requirement of Nse1, a subunit of the Smc5-Smc6 complex, for Rad52-dependent postreplication repair of UV-damaged DNA in Saccharomyces cerevisiae. Mol. Cell. Biol. 27, 8409-8418. doi: 10.1128/MCB.01543-07

Schlacher, K., Christ, N., Siaud, N., Egashira, A., Wu, H., and Jasin, M. (2011). Double-strand break repair-independent role for BRCA2 in blocking stalled replication fork degradation by MRE11. Cell 145, 529-542. doi: 10.1016/j.cell.2011.03.041

Schlacher, K., Wu, H., and Jasin, M. (2012). A distinct replication fork protection pathway connects Fanconi anemia tumor suppressors to RAD51-BRCA1/2. Cancer Cell 22, 106-116. doi: 10.1016/j.ccr.2012.05.015

Sen, D., Nandakumar, D., Tang, G. Q., and Patel, S. S. (2012). Human mitochondrial DNA helicase TWINKLE is both an unwinding and annealing helicase. J. Biol. Chem. 287, 14545-14556. doi: 10.1074/jbc.M111.309468

Sengupta, S., Linke, S. P., Pedeux, R., Yang, Q., Farnsworth, J., Garfield, S. H., et al. (2003). BLM helicasedependent transport of p53 to sites of stalled DNA replication forks modulates homologous recombination. EMBO J. 22, 1210-1222. doi: 10.1093/emboj/ cdg114

Sengupta, S., Robles, A. I., Linke, S. P., Sinogeeva, N. I., Zhang, R., Pedeux, R., et al. (2004). Functional interaction between BLM helicase and 53BP1 in a Chk1-mediated pathway during S-phase arrest. J. Cell Biol. 166, 801-813. doi: 10.1083/jcb.200405128

Sidorova, J. M., Kehrli, K., Mao, F., and Monnat, R. Jr. (2013). Distinct functions of human RECQ helicases WRN and BLM in replication fork recovery and progression after hydroxyurea-induced stalling.
DNA Repair 12, 128-139. doi: 10.1016/j.dnarep.2012.11.005

Singh, T. R., Ali, A. M., Busygina, V., Raynard, S., Fan, Q., Du, C. H., et al. (2008). BLAP18/RMI2, a novel OB-fold-containing protein, is an essential component of the Bloom helicase-double Holliday junction dissolvasome. Genes Dev. 22, 2856-2868. doi: 10.1101/gad.1725108

Sleeth, K. M., SØrensen, C. S., Issaeva, N., Dziegielewski, J., Bartek, J., and Helleday, T. (2007). RPA mediates recombination repair during replication stress and is displaced from DNA by checkpoint signalling in human cells. J. Mol. Biol. 373, 38-47. doi: 10.1016/j.jmb.2007.07.068

Thorslund, T., McIlwraith, M. J., Compton, S. A., Lekomtsev, S. Petronczki, M., Griffith, J. D., et al. (2010). The breast cancer tumor suppressor BRCA2 promotes the specific targeting of RAD51 to single-stranded DNA. Nat. Struct. Mol. Biol. 17, 1263-1265. doi: 10.1038/nsmb. 1905

Torres-Rosell, J., Sunjevaric, I., De Piccoli, G., Sacher, M., EckertBoulet, N., Reid, R., et al. (2007). The Smc5-Smc6 complex and SUMO modification of Rad52 regulates recombinational repair at the ribosomal gene locus. Nat. Cell Biol. 9, 923-931. doi: 10.1038/ncb1619

Ulrich, H. D. (2009). Regulating post-translational modifications of the eukaryotic replication clamp PCNA. DNA Repair 8, 461-469. doi: 10.1016/j.dnarep.2009.01.006

Vyas, R., Kumar, R., Clermont, F., Helfricht, A., Kalev, P., Sotiropoulou, P., et al. (2013). RNF4 is required for DNA doublestrand break repair in vivo. Cell Death Differ. 20, 490-502. doi: 10.1038/cdd.2012.145

Wechsler, T., Newman, S., and West, S. C. (2011). Aberrant chromosome morphology in human cells defective for Holliday junction resolution. Nature 471, 642-646. doi: 10.1038 /nature09790

Wray, J., Liu, J., Nickoloff, J. A., and Shen, Z. (2008). Distinct RAD51 associations with RAD52 and BCCIP in response to DNA damage and replication stress. Cancer Res. 68, 2699-2707. doi: 10.1158/0008-5472.CAN-07-6505

Wu, L., and Hickson, I. D. (2003). The Bloom's syndrome helicase suppresses crossing over during homologous recombination. Nature 426, 870-874. doi: 10.1038/nature02253

Wu, L., Bachrati, C. Z., Ou, J., Xu, C., Yin, J., Chang, M., et al. (2006). BLAP75/RMI1 promotes the BLM-dependent dissolution of homologous recombination intermediates. Proc. Natl. Acad. Sci. U.S.A. 103, 4068-4073. doi: 10.1073/pnas.0508295103

Wu, Y., Kantake, N., Sugiyama, T., and Kowalczykowski, S. C. (2008). Rad51 protein controls Rad52mediated DNA annealing. J. Biol. Chem. 283, 14883-14892. doi: 10.1074/jbc.M801097200

Xia, F., Taghian, D. G., DeFrank, J. S., Zeng, Z. C., Willers, H., Iliakis, G., et al. (2001). Deficiency of human BRCA2 leads to impaired homologous recombination but maintains normal nonhomologous end joining. Proc. Natl. Acad. Sci. U.S.A. 98, 8644-8649. doi: 10.1073/pnas. 151253498

Xu, D., Guo, R., Sobeck, A., Bachrati, C. Z., Yang, J., Enomoto, T., et al. (2008). RMI, a new OB-fold complex essential for Bloom syndrome protein to maintain genome stability. Genes Dev. 22, 2843-2855. doi: 10.1101/gad.1708608

Yamaguchi-Iwai, Y., Sonoda, E., Buerstedde, J. M., Bezzubova, O., Morrison, C., Takata, M., et al. (1998). Homologous recombination, but not DNA repair, is reduced in vertebrate cells deficient in RAD52. Mol. Cell. Biol. 18, 6430-6435.

Yang, H., Li, Q., Fan, J., Holloman, W. K., and Pavletich, N. P. (2005). The BRCA2 homologue Brh2 nucleates RAD51 filament formation at a dsDNA-ssDNA junction. Nature 433, 653-657. doi: 10.1038/nature03234

Yang, K., Moldovan, G. L., Vinciguerra, P., Murai, J., Takeda, S., and D'Andrea, A. D. (2011). Regulation of the Fanconi anemia pathway by a SUMO-like delivery network. Genes Dev. 25, 1847-1858. doi: 10.1101/gad.17020911

Yin, Y., Seifert, A., Chua, J. S., Maure, J. F., Golebiowski, F., and Hay, R. T. (2012). SUMO-targeted ubiquitin E3 ligase RNF4 is required for the response of human cells to DNA damage. Genes Dev. 26, 1196-1208. doi: $10.1101 /$ gad.189274.112

Yuan, J., Ghosal, G., and Chen, J. (2009). The annealing helicase HARP protects stalled replication forks. Genes Dev. 23, 2394-2399. doi: 10.1101/gad.1836409

Yusufzai, T., and Kadonaga, J. T. (2010). Annealing helicase 2 (AH2), a DNA-rewinding motor with an HNH motif. Proc. Natl. Acad. Sci. U.S.A. 107, 20970-20973. doi: 10.1073/pnas. 1011196107

Yusufzai, T., and Kadonaga, J. T. (2008). HARP is an ATP-driven annealing 
helicase. Science 322, 748-750. doi: 10.1126/science.1161233

Zhu, J., Zhu, S., Guzzo, C. M., Ellis, N. A., Sung, K. S., Choi, C. Y., et al. (2008). Small ubiquitinrelated modifier (SUMO) binding determines substrate recognition and paralog-selective SUMO modification. J. Biol. Chem. 283, 29405-29415. doi: 10.1074/jbc.M803632200
Conflict of Interest Statement: The authors declare that the research was conducted in the absence of any commercial or financial relationships that could be construed as a potential conflict of interest.

Received: 15 April 2013; accepted: 12 August 2013; published online: 04 September 2013.
Citation: Ouyang KJ, Yagle $M K$, Matunis MJ and Ellis NA (2013) BLM SUMOylation regulates ssDNA accumulation at stalled replication forks. Front. Genet. 4:167. doi: 10.3389/fgene. 2013.00167

This article was submitted to Cancer Genetics, a section of the journal Frontiers in Genetics.

Copyright @ 2013 Ouyang, Yagle, Matunis and Ellis. This is an open-access article distributed under the terms of the Creative Commons Attribution License (CC BY). The use, distribution or reproduction in other forums is permitted, provided the original author(s) or licensor are credited and that the original publication in this journal is cited, in accordance with accepted academic practice. No use, distribution or reproduction is permitted which does not comply with these terms. 